\title{
ROTURA YATRÓGENA DE URÉTER SECUNDARIA A LITOTRICIA EXTRACORPÓREA POR ONDAS DE CHOQUE
}

\author{
A. RODRÍGUEZ ALONSO, G. SUÁREZ PASCUAL, A. GONZÁLEZ BLANCO, \\ C. BONELLI MARTÍN, J. LORENZO FRANCO, M.Á. CUERPO PÉREZ, \\ Mํ.M. USED AZNAR*, J.C. ÁLVAREZ FERNÁNDEZ*, J. NIETO GARCÍA
}

Servicio de Urología. *Servicio de Anatomía Patológica. Hospital Arquitecto Marcide. El Ferrol (La Coruña).

Actas Urol Esp. 28 (7): 530-534, 2004

\section{RESUMEN \\ ROTURA YATRÓGENA DE URÉTER SECUNDARIA A LITOTRICIA EXTRACORPÓREA POR ONDAS DE CHOQUE}

La litotricia extracorpórea por ondas de choque es el tratamiento de elección de la mayoría de los cálculos urinarios, estimándose que sólo en el $5 \%$ de los casos es necesario recurrir a técnicas de cirugia abierta.

Las complicaciones derivadas de esta técnica son, en la mayoría de los casos, leves, transitorias y con escasa repercusión clínica, observándose sólo en casos aislados lesiones urológicas o extraurológicas de gravedad. Dichas complicaciones se producen bien por la propia acción del cálculo o sus fragmentos, o bien por la acción de las ondas de choque.

Presentamos un caso de rotura ureteral secundaria al tratamiento de un cálculo ureteral mediante litotricia extracorpórea por ondas de choque, que derivó en un absceso retroperitoneal periureteral, y fue tratado mediante nefroureterectomía.

PALABRAS CLAVE: Litotricia. Complicaciones. Rotura de uréter. Litiasis urinaria.

\section{ABSTRACT \\ IATROGENIC RUPTURE OF THE URETER FOLLOWING EXTRACORPOREAL SHOCK WAVE LITHOTRIPSY}

Extracorporeal shock wave lithotripsy is the treatment of choice in most cases of urinary calculi, with the estimation that open surgery is only necessary in 5\% of all cases.

In most cases, the complications derived from this technique are slight, transitory and of little clinical importance, with isolated cases of serious urological or extraurological injury. These complications are either caused by the action of the stone itself or its fragments, or by the action of shock waves.

We present a case of ureteral rupture following treatment of a ureteral calculus using extracorporeal shock wave lithotripsy which led to a periureteral retroperitoneal abscess that was treated by nephroureterectomy.

KEY WORDS: Lithotripsy. Complications. Ureter rupture. Urinary lithiasis. 
$\mathrm{E}_{\mathrm{p}}^{\mathrm{n}}$ 1980 Chaussy et al. realizan con éxito el primer tratamiento de litiasis urinaria mediante litotricia extracorpórea por ondas de choque (LEOC), en la Universidad de Munich. En aquel momento, los autores limitaron las indicaciones de esta nueva técnica al 15\% de los cálculos urinarios ${ }^{1}$. A partir de 1982 la LEOC se utiliza de forma rutinaria en el tratamiento de la litiasis urinaria ${ }^{2}$.

La LEOC es en la actualidad el tratamiento de elección de la mayoría de los cálculos localizados en cualquier segmento del tracto urinario superior $^{2}$. Se estima que el 90-95\% de los cálculos urinarios pueden ser eliminados satisfactoriamente mediante la utilización de LEOC, nefrolitotomía percutánea y ureterorrenoscopia, solas o en combinación ${ }^{1}$. Sólo en el 5\% de los casos es necesario recurrir a técnicas de cirugía abierta ${ }^{3,4}$.

La LEOC no es una técnica exenta de complicaciones. En la mayoría de los casos las complicaciones son leves y transitorias, pero es importante conocer su existencia para tratarlas precozmente ${ }^{1,2}$.

Presentamos un caso de rotura yatrógena de uréter ocasionada por LEOC, que se complicó con un absceso retroperitoneal y fue tratado mediante nefroureterectomía.

\section{CASO CLÍNICO}

Mujer de 72 años, con antecedentes de diabetes mellitus no insulín-dependiente e hipertensión arterial, que ingresó de forma urgente en el servicio de Urología por cólico nefrítico derecho complicado.

La paciente era seguida en la consulta externa por historia de litiasis urinaria. Presentaba un cálculo radiopaco de aproximadamente $3 \mathrm{~cm}$ en pelvis renal derecha, que fue tratado mediante LEOC (4500 ondas de choque a 15,7 kV), consiguiendo una fragmentación parcial y expulsión de pequeños fragmentos litiásicos (Fig. 1).

En el estudio de control post-LEOC, se comprobaba la disminución de tamaño $(1,5 \mathrm{~cm}) \mathrm{y}$ el descenso del cálculo, que en ese momento se localizaba en el tercio proximal del uréter derecho, realizándose nueva sesión de LEOC (5000 ondas de choque a $18,7 \mathrm{kV}$ ) (Fig. 2).

Diez días después de la última LEOC, la paciente acudía al Servicio de Urgencias por cuadro de 3

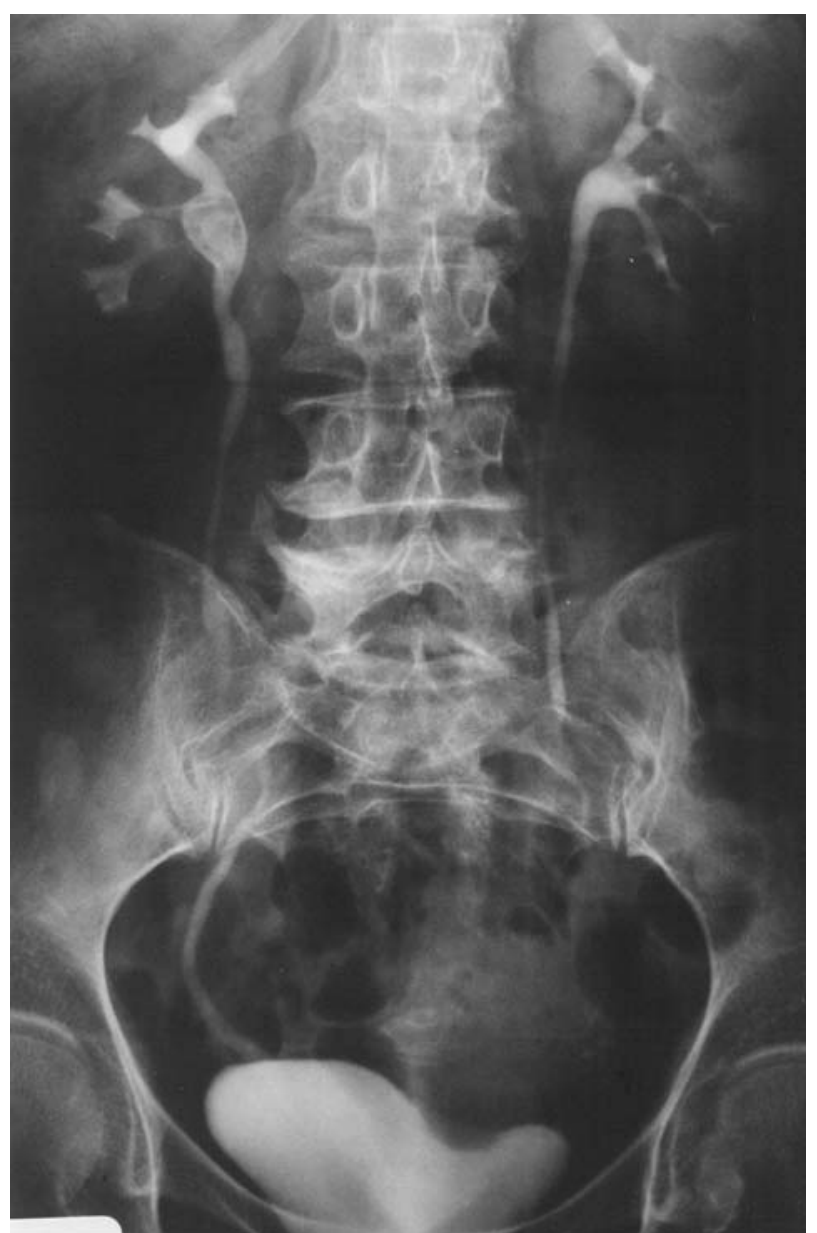

FIGURA 1. UIV en la que se observa un cálculo piélico derecho de aproximadamente $3 \mathrm{~cm}$, con ligera ectasia infundibulo-calicial.

días de evolución consistente en dolor lumbar derecho, fiebre vespertina $>38^{\circ} \mathrm{C}$, astenia, náuseas y vómitos.

A la exploración física presentaba dolor a la puño-percusión renal derecha, así como dolor y empastamiento en fosa iliaca y vacío derechos, con importante defensa muscular.

Las pruebas de laboratorio al ingreso mostraban los siguientes datos: hemograma: $\mathrm{Hb}$ 10,7 g/dl, Hcto 31,7\%, VCM 78,2 fl, HbCM 27,2 pg, leucocitos $18200 / \mathrm{ml}$ con $81 \%$ de neutrófilos. Bioquímica sanguínea: glucosa $192 \mathrm{mg} / \mathrm{dl}$, urea $71 \mathrm{mg} / \mathrm{dl}$, creatinina 1,8 mg/dl. Coagulación: normal. Sedimento de orina: piuria y bacteriuria intensas, con pH 6. Cultivo de orina: E. coli.

Con el diagnóstico de cólico nefrítico complicado se realizó una ecografía abdominal en la que se evidenciaba ureterohidronefrosis derecha y 


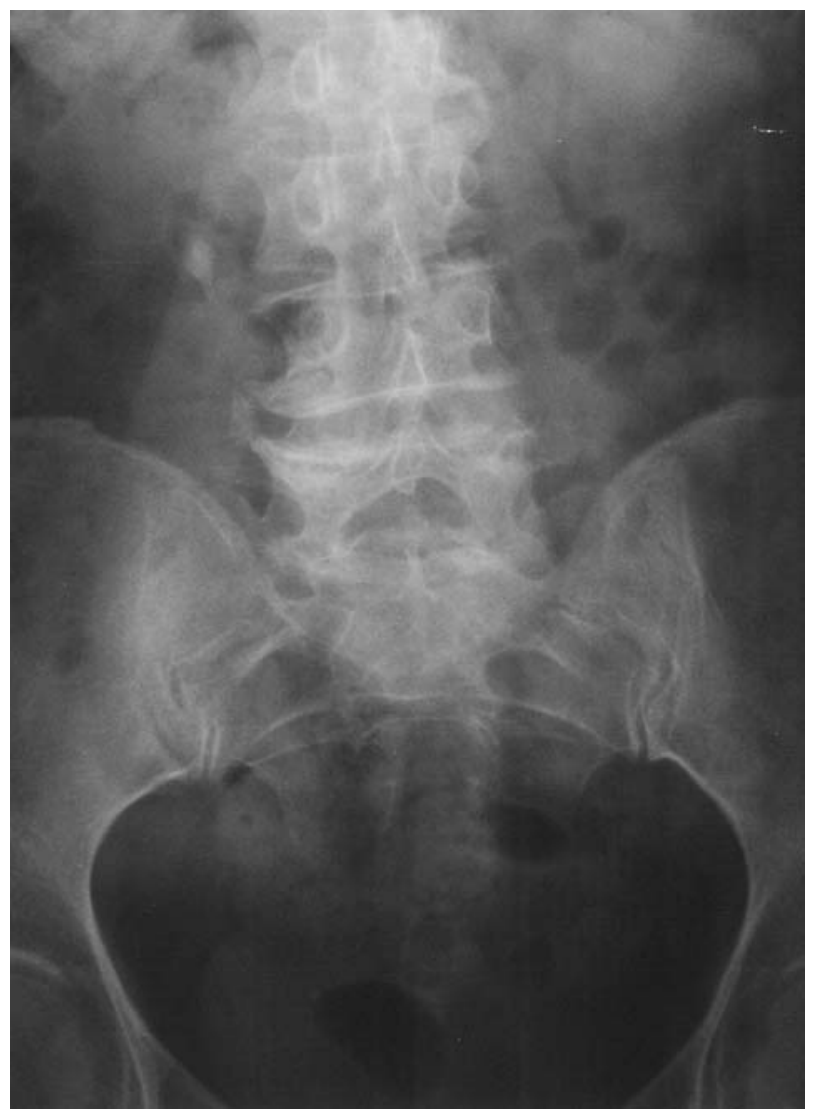

FIGURA 2. Radiografia simple de abdomen tras la primera LEOC, en la que se observa descenso del cálculo y disminución de su tamaño.

engrosamiento segmentario del músculo psoas iliaco. Se realizó asimismo una UIV, en la que se observaba cálculo radiopaco multifragmentado de aproximadamente $1,5 \mathrm{~cm}$, proyectándose sobre la pala iliaca derecha, borramiento de la línea del psoas derecho y anulación funcional del riñón derecho, siendo el resto del aparato urinario morfológica y funcionalmente normal.

Ante estos hallazgos, se realizó intubación ureteral con catéter doble $\mathrm{J}$, observando dificultad de progresión del mismo a partir de $15 \mathrm{~cm}$ del meato ureteral derecho.

A continuación se realizó TAC abdomino-pélvico, observando riñón derecho ligeramente atrófico con uropatía obstructiva ipsilateral (Fig. 3). Se demostraba asimismo catéter endoureteral, cuyo extremo craneal se introducía a través de una rotura de la pared ureteral posterior, en el seno de una masa ubicada entre el uréter y el músculo psoas iliaco, con características densitométricas de absceso (Fig. 4).

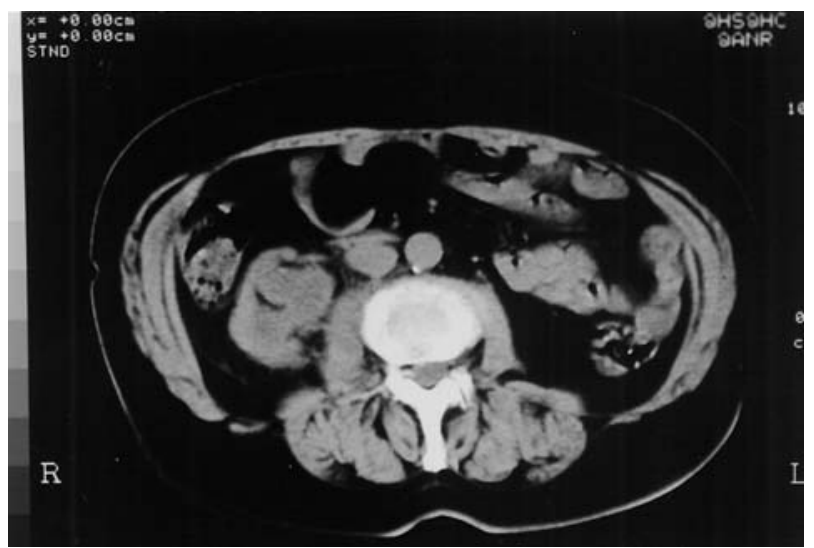

FIGURA 3. TAC abdominal en el que se observa hidronefrosis derecha con marcada atrofia cortical.

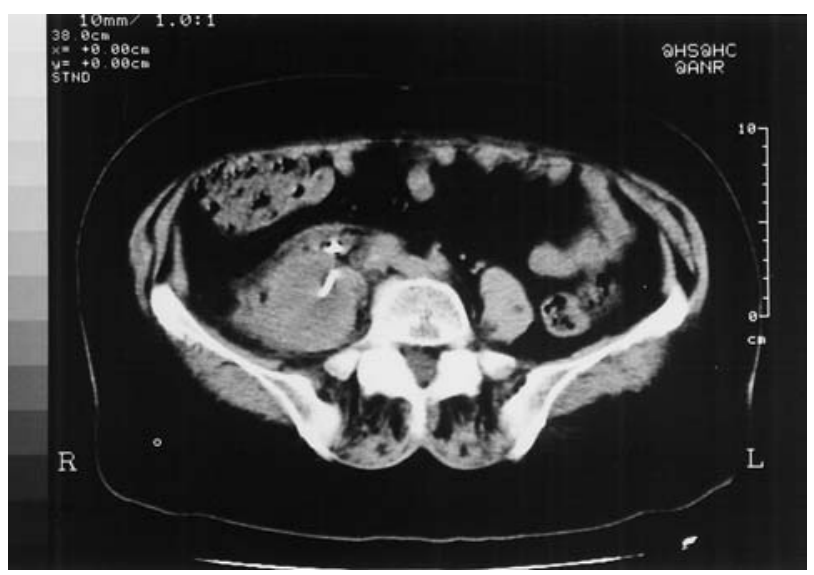

FIGURA 4. TAC abdominal en el que se observa un absceso situado entre el uréter derecho y el músculo psoas. Catéter ureteral introduciéndose en el interior del absceso tras atravesar la pared ureteral posterior.

Se decidió efectuar pielografía ascendente derecha, demostrándose ausencia de relleno del tercio proximal del uréter y extravasación de contraste al espacio retroperitoneal.

En esta fase la paciente se encontraba postrada, presentando una temperatura de $39^{\circ} \mathrm{C}$, con escalofríos y una tensión arterial inestable con máximos de 80/50 mmHg. Por ello se decidió realizar intervención quirúrgica, practicando nefroureterectomía derecha, con drenaje y limpieza de absceso retroperitoneal interurétero-psoico (Fig. 5).

El cultivo del material purulento retroperitoneal fue positivo para E. coli. En el estudio anatomopatológico se observaba pielonefritis abscesificada sobre riñón con pielonefritis crónica, esclerosis y atrofia incompleta. Múltiples fragmentos litiásicos intraureterales. Cálculo multifragmentado en tercio ureteral distal (Fig. 6). 
El post-operatorio transcurrió con normalidad, y la paciente fue dada de alta al séptimo día.

Dieciocho meses después de la intervención quirúrgica, la paciente se encuentra asintomática, con una creatinina de $1,5 \mathrm{mg} / \mathrm{dl}$ y un buen funcionalismo del tracto urinario superior izquierdo, según se demuestra mediante urografía intravenosa.

\section{DISCUSIÓN}

Las complicaciones derivadas del tratamiento de los cálculos urinarios mediante LEOC pueden clasificarse en: complicaciones ocasionadas por

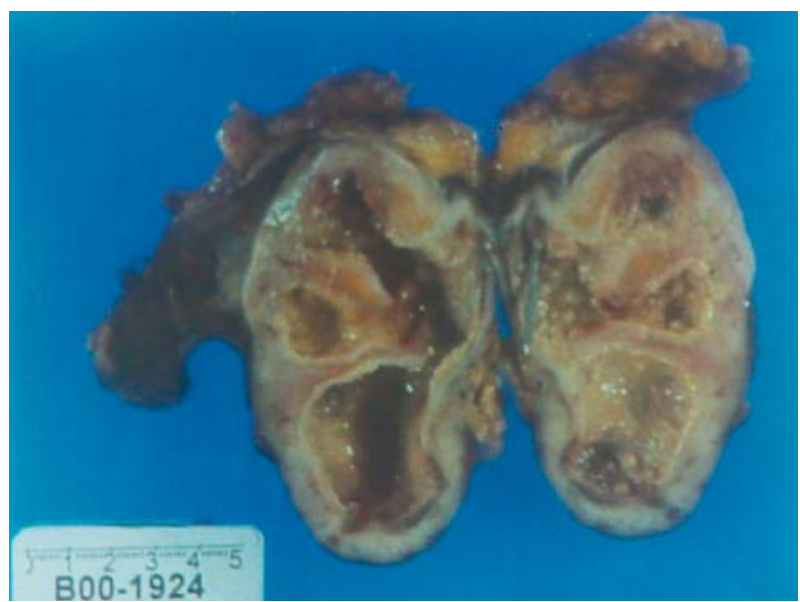

FIGURA 5. Pieza de nefroureterectomía en la que se aprecia un riñón pielonefritico crónico, con atrofia cortical e importante dilatación pielocalicial.

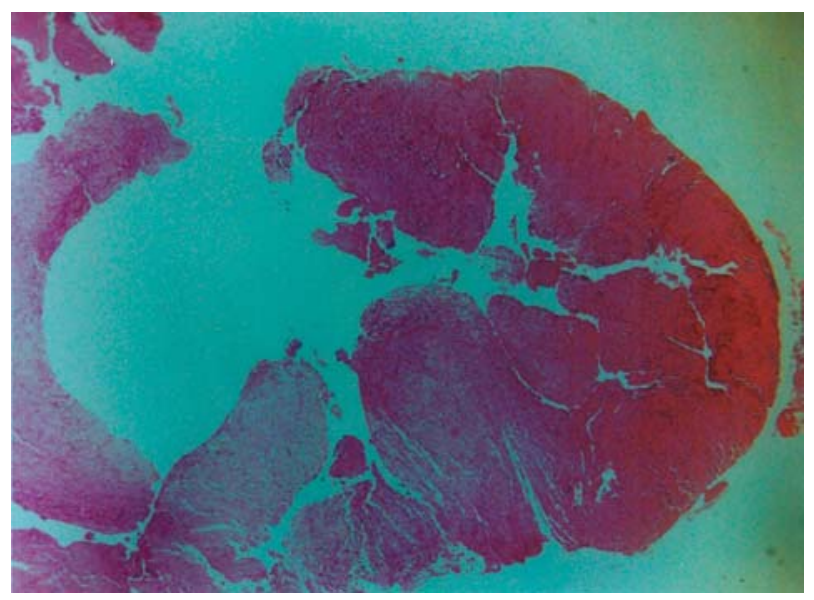

FIGURA 6. Corte histológico del uréter a la altura del absceso periureteral (10X) en el que se aprecia un área de completa pérdida de continuidad de la pared, infiltración inflamatoria y ausencia total de epitelio transicional. la acción del cálculo o sus fragmentos y complicaciones ocasionadas por la acción de las ondas de choque. En muchas ocasiones la complicación es una consecuencia de la interacción de ambos mecanismos de acción.

La complicación más frecuentemente observada es la hematuria, que se produce en más del 80\% de los casos, considerándose más un hallazgo normal que una verdadera complicación ${ }^{3-5}$. El cólico nefrítico se produce en el 10-25\% de los casos, según las series, y es consecuencia de la obstrucción ureteral ocasionada por fragmentos o por calle litiásica ${ }^{1,4,5}$. Se observa asimismo sintomatología irritativa del tracto urinario inferior en el 38\% de las ocasiones, cuando el cálculo o sus fragmentos se sitúan en el uréter yuxtavesical o intramural ${ }^{4}$. Es poco frecuente el desarrollo de hematomas renales (parenquimatoso, subcapsular o perirrenal) clínicamente significativos, sin embargo en algunos estudios se ha demostrado un hematoma renal en el 29\% de los pacientes a los que se les realiza una Resonancia Magnética tras la LEOC ${ }^{5,6}$. Otras complicaciones menos frecuentes son: sepsis urinaria $(0,4-2,3 \%)$ y anuria $(1,2 \%)^{1,4}$.

La acción de las ondas de choque puede producir lesiones de órganos adyacentes al aparato urinario, aunque la frecuencia de esta complicación es muy baja $(0,3 \%)^{4}$. Algunos de los órganos que pueden resultar lesionados por la LEOC son: intestino delgado y colon (melenas), páncreas (pancreatitis aguda) e hígado (hipertransaminemia) $)^{6,7}$. En general estas complicaciones muestran escasa repercusión clínica y se resuelven satisfactoriamente con tratamiento conservador.

Se han descrito también complicaciones de mayor gravedad, tanto en la esfera urológica: rotura de pelvis renal $^{8}$, como del ámbito extraurológico: rotura esplénica ${ }^{9}$ y rotura de aneurisma de aorta abdominal ${ }^{10}$.

Según Fuchs GJ et al. ${ }^{7}$ la mayoría de las complicaciones derivadas de la LEOC son consecuencia de: a) una indicación inapropiada; b) aplicación de una dosis demasiado elevada de ondas de choque; c) una vigilancia inadecuada de los pacientes tras la LEOC; o d) un desconocimiento de las técnicas endourológicas auxiliares. 
La rotura del uréter en pacientes tratados con LEOC es una complicación de presentación muy infrecuente. Dicha lesión puede ser una consecuencia de la acción directa de las ondas de choque sobre la pared ureteral o bien de la acción indirecta de los fragmentos litiásicos, según discuten Alkibay T et al., en un caso de rotura piéli$\mathrm{ca}^{8}$. En nuestro caso, el incremento de la presión ureteral ocasionado por algún fragmento litiásico, actuando sobre el sustrato de una pared ureteral debilitada y lesionada, pudo desencadenar una rotura ureteral, con formación de un urinoma periureteral.

El hallazgo radiológico de un absceso retroperitoneal, secundario a la sobreinfección de un urinoma, observado en la paciente de nuestro caso, hacía necesario un drenaje urgente de dicha colección.

El estado de pre-shock, con clínica séptica e inestabilidad hemodinámica significativa, en una paciente de riesgo elevado por su patología diabética subyacente, nos inclinó a realizar un abordaje quirúrgico abierto. El mal estado del uréter, con una extensa lesión de su pared y áreas de isquemia y necrosis, y la atrofia y retracción pielonefrítica del parénquima renal nos hicieron optar por una nefroureterectomía.

\section{REFERENCIAS}

1. PÉREZ HERMS S, CASTELLANOS ACOSTA R, CORTADELLAS ÁNGEL R, GUZMÁN FERNÁNDEZ A, BALLESTEROS SAMPOL JJ.: Nefrectomía tras tratamiento con ESWL de una litiasis coraliforme complicada con urinoma. Arch Esp Urol 1992; 45 (7): 691-693.
2. AUGE BK, PREMINGER GM.: Update on shock wave lithotripsy technology. Curr Opin Urol 2002; 12: $287-290$.

3. YAGODA SHAGAM J.: Extracorporeal shock wave lithotripsy. Radiol Technol 2000; 72 (2): 145-167.

4. GONZÂLEZ ENGUITA C, CABRERA PÉREZ J, CALAHORRA FERNÁNDEZ FJ, CANCHO GIL MJ, VELA NAVARRETE R.: Tratamiento de la litiasis ureteral con ondas de choque. Arch Esp Urol 2001; 54 (9): 971-982.

5. ATALA A, STEINBOCK GS.: Extracorporeal shockwave lithotripsy of renal calculi. Am J Surg 1989; 157: 350-358.

6. BUSH WH: Radiology and treatment of urinary tract stone disease. Curr Opin Radiol 1992; 4: 3238.

7. FUCHS GJ, DAVID RD, FUCHS AM.: Complicaciones de la litotricia extracorpórea por ondas de choque. Arch Esp Urol 1989; 42 (supl 1): 83-89.

8. ALKIBAY T, KARAOGLAN Ü, GÜNDOGDU S, BOZKIRLI I.: An unusual complication of extracorporeal shock wave lithotripsy: urinoma due to rupture of the renal pelvis. Int Urol Nephrol 1992; 24 (1): 11-14.

9. FUGITA OEH, TRIGO-ROCHA F, MITRE AI, ARAP S.: Splenic rupture and abscess after extracorporeal shock wave lithotripsy. Urology 1998; 52 (2): 322-323.

10. LAZARIDES MK, DRISTA H, ARVANITIS DP, DAYANTAS JN.: Aortic aneurysm rupture after extracorporeal shock wave lithotripsy. Surgery 1997; 122: 112-113.

Dr. A. Rodríguez Alonso

(Servicio de Urologia)

Hospital Arquitecto Marcide

Ctra. San Pedro de Leixa, s/n

15405 El Ferrol (La Coruña)

(Trabajo recibido el 3 julio de 2003) 